UTTG-11-92

\title{
Three-Body Interactions Among Nucleons and Pions
}

\author{
Steven Weinberg用 \\ Theory Group \\ Department of Physics \\ University of Texas \\ Austin, Texas 78712
}

\begin{abstract}
A chiral invariant effective Lagrangian may be used to calculate the three-body interactions among low-energy pions and nucleons in terms of known parameters. This method is illustrated by the calculation of the pion-nucleus scattering length.
\end{abstract}

* Research supported in part by the Robert A. Welch Foundation and NSF Grant PHY 9009850 . 
Recent articles $^{1,2}$ have described a systematic effective Lagrangian framework for the calculation of reactions involving arbitrary numbers of nucleons as well as pions of low 3-momentum. To leading order in small momenta, the 'potential' for such reactions is given entirely by the tree graphs in which only two of the pions and/or nucleons interact; further, their interaction is calculated using the original effective chiral Lagrangian ${ }^{3}$, which consists of terms with only the minimum numbers of derivatives or pion mass factors, supplemented by contact interaction terms among nucleons. The corrections to these two-body interactions of second order in small momenta involve not only one-loop graphs, but also a large number of new terms ${ }^{4}$ in the Lagrangian with additional derivatives, so many that not much can be learned about pion-nucleon or nucleon-nucleon interactions in this way. Fortunately, these two-body interactions can instead be taken from phenomenological models that incorporate experimental information on nucleon-nucleon, pionnucleon, and pion-pion scattering. The only remaining contributions to the potential of the same order in small momenta consist of graphs in which three particles (or two pairs of particles) interact, their interactions given by tree graphs calculated from the original effective chiral Lagrangian. Thus we can use the three-body interactions calculated in terms of known parameters from the original effective chiral Lagrangians together with experimental data on two-body scattering to calculate all corrections to the potential of first and second order in small momenta. 
This method will be illustrated here in the calculation of the amplitudes for pion scattering on complex nuclei. But first, a reminder of some generalities.

Consider the amplitude for a process with $N_{n}$ nucleons and $N_{\pi}$ pions in the initial state and the same numbers of nucleons and pions in the final state, all with 3-momenta no larger than of order $m_{\pi}$. We wish to develop a perturbation theory for this amplitude, based on an expansion in powers of the ratio of these small momenta (and the pion mass) to some momentum scale that is characteristic of quantum chromodynamics, such as $m_{\rho}$. In counting the number of powers of small momenta in any given "old fashioned" (time-ordered) diagram for this process, we must distinguish between energy denominators of two types. Those of the first type arise from intermediate states that differ from the initial and final states in the number of pions and/or in the pion energies, and are therefore of the order of the small momenta or the pion mass. The energy denominators of the second type arise from intermediate states that differ from the initial and final states only in the nucleon momenta, and are therefore much smaller, of the order of the nucleon kinetic energies. A given graph is called irreducible if it contains only energy denominators of the first type. These are graphs for which the initial particle lines cannot all be disconnected from the final particle lines by cutting through any intermediate state containing $N_{n}$ nucleons and either all the initial or all the final pions. We shall consider disconnected as well 
as connected irreducible graphs, because a general connected graph is built up from a sequence of both disconnected and connected irreducible graphs interleaved with small energy denominators of the second type. (However in all graphs considered here, each of the initial particle lines must be connected to one or more of the final particle lines, and vice versa.) This sum of disconnected and connected irreducible graphs is what was referred to above as the potential.

Because irreducible graphs do not contain anomalously small energy denominators of the order of nucleon kinetic energies, it is easy to count the number $\nu$ of powers of small momenta or pion masses in these graphs. For an irreducible graph with $V_{i}$ vertices of type $i, L$ loops, and $C$ separate connected pieces, the number of powers of small momenta or pion masses is ${ }^{1,2}$

$$
\nu=4-N_{n}-2 C+2 L+\sum_{i} V_{i} \Delta_{i}
$$

where $\Delta_{i}$ is an index for an interaction of type $i$, given in terms of the number $n_{i}$ of nucleon field factors and the number $d_{i}$ of derivatives (or powers of pion mass) in the interaction, by

$$
\Delta_{i}=d_{i}+\frac{1}{2} n_{i}-2
$$

(In deriving this result, we count -3 powers of small momenta for each line passing without interaction through the diagram, because the associated momentum-space delta function reduces the number of momentum factors in the total connected amplitude by that amount.) 
Eq. (1) is useful because chiral invariance rules out any terms in the Lagrangian with $\Delta_{i}<0$. It follows that for any given number of external lines, the leading irreducible graphs (those with smallest $\nu$ ) are the tree graphs (i. e., $L=0$ ) with the maximum number $C$ of connected parts, constructed solely from vertices with $\Delta_{i}=0$. The contribution of these vertices can be read off from the effective interaction Hamiltonian (in the interaction picture):

$$
\begin{aligned}
H_{\mathrm{int}, \Delta=0}= & \frac{1}{2}\left(D^{2}-1\right) \dot{\boldsymbol{\pi}}^{2}+\frac{1}{2}\left(D^{-2}-1\right) \vec{\nabla} \boldsymbol{\pi} \cdot \vec{\nabla} \boldsymbol{\pi}+\frac{1}{2} m_{\pi}^{2}\left(D^{-1}-1\right) \boldsymbol{\pi}^{2} \\
& +2 F_{\pi}^{-4}(\bar{N}(\boldsymbol{t} \times \boldsymbol{\pi}) N)^{2} \\
& +\bar{N}\left[2 F_{\pi}^{-1} g_{A} D^{-1} \boldsymbol{t} \cdot(\vec{\sigma} \cdot \vec{\nabla} \boldsymbol{\pi})+2 F_{\pi}^{-2} D \boldsymbol{t} \cdot(\boldsymbol{\pi} \times \dot{\boldsymbol{\pi}})\right] N \\
& +\frac{1}{2} C_{S}(\bar{N} N)(\bar{N} N)+\frac{1}{2} C_{T}(\bar{N} \vec{\sigma} N)(\bar{N} \vec{\sigma} N)
\end{aligned}
$$

which is derived from the most general chiral-invariant Lagrangian with $\Delta_{i}=$ 0 :

$$
\begin{aligned}
\mathcal{L}_{\Delta=0}= & -\frac{1}{2} D^{-2} \partial_{\mu} \boldsymbol{\pi} \cdot \partial^{\mu} \boldsymbol{\pi}-\frac{1}{2} D^{-1} m_{\pi}^{2} \boldsymbol{\pi}^{2} \\
& +\bar{N}\left[i \partial_{0}-2 D^{-1} F_{\pi}^{-2} \boldsymbol{t} \cdot\left(\boldsymbol{\pi} \times \partial_{0} \boldsymbol{\pi}\right)-m_{N}-2 D^{-1} F_{\pi}^{-1} g_{A} \boldsymbol{t} \cdot(\vec{\sigma} \cdot \vec{\nabla}) \boldsymbol{\pi}\right] N \\
& -\frac{1}{2} C_{S}(\bar{N} N)(\bar{N} N)-\frac{1}{2} C_{T}(\bar{N} \vec{\sigma} N) \cdot(\bar{N} \vec{\sigma} N)
\end{aligned}
$$

where $g_{A} \simeq 1.25$ and $F_{\pi} \simeq 190 \mathrm{MeV}$ are the usual axial coupling constant and pion decay amplitude; $\boldsymbol{t}$ is the nucleon isospin matrix; $C_{S}$ and $C_{T}$ are constants whose values can be inferred from the singlet and triplet neutronproton scattering lengths; and $D \equiv 1+\pi^{2} / F_{\pi}^{2}$. (As discussed in ref. 2 , terms 
involving time-derivatives of the nucleon field are eliminated by a suitable redefinition of that field, while corrections to the non-relativistic treatment of the nucleon in (3) and (4) appear as terms in the effective Hamiltonian and Lagrangian with $\Delta_{i}>0$.) The number $C$ of connected parts is given its maximum value $C=N_{n}+N_{\pi}-1$ by including only graphs (with one qualification to be discussed later) for a single $\pi N, N N$, or $\pi \pi$ scattering, with all other lines passing without interaction through the diagram.

The corrections to these leading terms with only one extra factor of small momenta (or $m_{\pi}$ ) arise from (a) tree graphs, with the maximum number $C=N_{n}+N_{\pi}-1$ of connected parts, that involve a single vertex (such as those arising from non-zero $u$ and $d$ quark masses) with $\Delta_{i}=1$, plus any number of vertices with $\Delta_{i}=0$. The next corrections, with two extra factors of small momenta (or $m_{\pi}$ ), arise from (b) one-loop graphs with $C=N_{n}+N_{\pi}-1$ involving only vertices with $\Delta_{i}=0$; (c) tree graphs with $C=N_{n}+N_{\pi}-1$ involving either two vertices with $\Delta_{i}=1$ or one vertex with $\Delta_{i}=2$ (which serve as counterterms for the infinities encountered in one-loop graphs), as well as any number of vertices with $\Delta_{i}=0 ;$ (d) tree graphs, constructed entirely from vertices with $\Delta_{i}=0$, that have one less than the maximum number of connected parts, i. e., with $C=N_{n}+N_{\pi}-2$.

As already mentioned, the vertices with $\Delta_{i}=2$ that contribute to corrections of type (c) contain so many free parameters ${ }^{4}$ that little of value can be learned by using the effective Lagrangian to calculate these corrections. 
On the other hand, these corrections as well as the leading terms and the corrections of types (a) and (b) all only contribute to the maximally disconnected irreducible graphs, that consist of a connected piece involving just two of the incoming nucleons and/or pions, plus disconnected lines passing without interaction through the diagram for all of the other incoming nucleons and pions. But instead of trying to use the effective Lagrangian to calculate such two-body interactions, we can draw on various phenomenological models that incorporate not only chiral symmetry but the whole body of present experimental information about low energy nucleon-nucleon, nucleon-pion, and pion-pion scattering.

There remain only the corrections of type (d), with $C=N_{n}+N_{\pi}-2$. These are to be calculated from tree graphs involving only the $\Delta_{i}=0$ Hamiltonian (3), which involves no unknown parameters. These corrections arise from graphs that either consist of $(\mathrm{d} 1)$ a connected piece involving just three of the incoming nucleons and/or pions, or (d2) two connected pieces each involving just two of the incoming nucleons and/or pions, plus in both cases disconnected lines passing without interaction through the diagram for all of the other incoming nucleons and pions. Graphs of type (d2) may, like the graphs of types (a), (b), and (c), be taken from suitable phenomenological models based on experimental information about two-body scattering processes. This leaves only the three-body graphs of type (d1), which can be calculated from first principles in terms of known constants. 
Let's first see how this applies to processes involving only nucleons. Multinucleon scattering amplitudes and bound-state wave functions are found by solving an inhomogeneous Lippman-Schwinger or homogeneous Schrödinger equation with the effective potential taken as the sum of irreducible graphs. The graphs for the three-nucleon terms in the effective potential are shown in Figures (1) and (2). A cancellation (to leading order in small momenta) was noted in reference 2 among the graphs of Figure (1), the only graphs that involve the part of the effective Hamiltonian (3) that is non-linear in the pion field. It is instructive to look at the reason for this cancellation. These graphs all involve a single quadratic interaction $2 F_{\pi}^{-1} g_{A} \bar{N} \boldsymbol{t} \cdot(\boldsymbol{\pi} \times \dot{\boldsymbol{\pi}}) N$ plus linear interactions of the two pion fields in this interaction with the other two nucleons. In each individual time-ordered graph, the time derivative in the quadratic interaction makes a contribution of the order of a pion energy. However, by summing up the old-fashioned graphs for all the time-orderings of these three vertices, we obtain a Feynman diagram in which energy is conserved at each vertex, so that the time-derivative yields a difference of nucleon kinetic energies, smaller by a factor at most of order $m_{\pi} / m_{N}$.

This leaves the 3-nucleon graphs of Figure (2). These are genuine contributions to what we have defined as the 3-nucleon potential, but they involve only the contact and pion-exchange nucleon-nucleon interactions, and their effect is actually cancelled by terms in the expansion of the reducible threenucleon graphs of Figure (3) in powers of the ratio of nucleon to pion kinetic 
energies. Again, the reason for this cancellation is not hard to find. Although in Figure (2) we are not summing over all time orderings, so that these graphs do not make up a complete Feynman diagram, the sum of all the time-ordered graphs of Figures (2) and (3) makes up several complete Feynman diagrams, in which energy denominators are replaced with pion and nucleon propagators, and energy is conserved at each vertex. Since the virtual pion energies in these Feynman diagrams are equal to differences of nucleon kinetic energies, and hence negligible compared with the virtual pion 3-momenta $\vec{q}$, the pion propagators in these diagrams are just $\left(\vec{q}^{2}+m_{\pi}^{2}\right)^{-1}$. But these Feynman diagrams with such pion propagators are just what we would get from the old-fashioned diagrams of Figure (3) if we were to neglect nucleon kinetic energies in energy denominators for states containing a pion. Thus we may calculate the multi-nucleon potential to second order in small momenta by ignoring nucleon kinetic energies in the energy denominators of the leading pion-exchange contributions to the potential, and ignoring the three (or more) - nucleon contributions altogether. This is more or less what nuclear physicists have always done anyway.:.

The three-body forces are more interesting in processes involving a pion. For definiteness, consider the low-energy elastic scattering of a pion from a nucleus of nucleon number $A$. General considerations of scattering theory tell

*I am grateful to J. Friar for pointing out that in some treatments of the nuclear threebody problem the pion exchange forces are calculated neglecting nucleon kinetic energies in energy denominators, and that the corrections to this approximation are of the same order as the other corrections considered in this work. 
us that the S-matrix element for this process is simply given by the matrix element between nuclear wave functions of the sum of all irreducible graphs with $N_{n}=A$ and $N_{\pi}=1$. (In applying the effective chiral Lagrangian to such processes we are making use of the fact that typical 3-momenta of nucleons in nuclei are of order $m_{\pi}$ or less.) The leading irreducible graphs are those in which the pion scatters off a single nucleon, evaluated using the $\Delta_{i}=0$ vertices in the tree approximation.** To second order in small momenta, the corrections to these leading terms arise from corrections to the pion-nucleon scattering amplitude (from loop graphs and from vertices with $\left.\Delta_{i}=1,2\right)$ which can be taken from phenomonological models of pionnucleon scattering, together with connected three-body interactions among two nucleons and the pion, calculated from tree graphs evaluated with the $\Delta_{i}=0$ vertices in Eq. (3). The graphs for these three-body interactions are shown in Figure 4.

This is a lot to calculate, but the problem becomes much simpler if we restrict our attention to the pion-nucleus scattering length, for which the incoming and outgoing pion have vanishing 3-momenta. The leading terms as well as the corrections to pion-nucleon scattering give a scattering length that (apart from reduced-mass corrections) is just the sum of the scattering

\footnotetext{
** There are also nominally leading terms in which the incoming pion is absorbed by one nucleon and the outgoing pion is emitted by another, but when these are summed over different time-orderings they cancel. Again, this is because summing over time-orderings yields a Feynman diagram in which energy is conserved, but energy cannot be conserved in the emission or absorption of a single real pion by a single nucleon.
} 
lengths on the individual nucleons. This leaves only the three-body irreducible graphs, of which the only ones that survive in the limit of vanishing external pion 3-momenta are those shown in Figures 4(a) to 4(f).

It is easiest to calculate the contributions of Figures 4(a)-4(c) and 4(f) by noting that the sum over time orderings in graphs of each type [and lumping together graph $4(\mathrm{f})$, produced by the interaction term $2 F_{\pi}^{-4}(\bar{N}(\boldsymbol{t} \times \boldsymbol{\pi}) N)^{2}$ in the Hamiltonian (3), with the other graphs] must give the same result as the complete Feynman diagrams of type 4(a) - 4(c) calculated from the Lagrangian (4) [which does not contain the interaction $2 F_{\pi}^{-4}(\bar{N}(\boldsymbol{t} \times \boldsymbol{\pi}) N)^{2}$.] The other graphs, 4(d) and 4(e), are not summed over all time-orderings (because the sum would include reducible as well as irreducible graphs) and so their contributions must be calculated using old-fashioned perturbation theory. These contributions to the pion-nucleon scattering length are:

$$
\begin{aligned}
a_{a b}^{[4(a)]} & =\frac{m_{\pi}^{2}}{2 \pi^{4} F_{\pi}^{4}\left(1+m_{\pi} / m_{d}\right)} \sum_{r<s}\left\langle\frac{1}{\vec{q}_{r s}^{2}}\left(2 \boldsymbol{t}^{(r)} \cdot \boldsymbol{t}^{(s)} \delta_{a b}-t_{a}^{(r)} t_{b}^{(s)}-t_{a}^{(s)} t_{b}^{(r)}\right)\right\rangle \\
a_{a b}^{[4(b)]} & =-\frac{g_{A}^{2} \delta_{a b}}{2 \pi^{4} F_{\pi}^{4}\left(1+m_{\pi} / m_{d}\right)} \sum_{r<s}\left\langle\boldsymbol{t}^{(r)} \cdot \boldsymbol{t}^{(s)} \frac{\vec{q}_{r s} \cdot \vec{\sigma}^{(r)} \vec{q}_{r s} \cdot \vec{\sigma}^{(s)}}{\vec{q}_{r s}^{2}+m_{\pi}^{2}}\right\rangle, \\
a_{a b}^{[4(c)]} & =\frac{g_{A}^{2}}{2 \pi^{4} F_{\pi}^{4}\left(1+m_{\pi} / m_{d}\right)} \\
\times & \sum_{r<s}\left\langle\frac{\left[\vec{q}_{r s}^{2} \boldsymbol{t}^{(r)} \cdot \boldsymbol{t}^{(s)} \delta_{a b}+m_{\pi}^{2}\left(t_{a}^{(r)} t_{b}^{(s)}+t_{a}^{(s)} t_{b}^{(r)}\right)\right] \vec{q}_{r s} \cdot \vec{\sigma}^{(r)} \vec{q}_{r s} \cdot \vec{\sigma}^{(s)}}{\left(\vec{q}_{r s}^{2}+m_{\pi}^{2}\right)^{2}}\right\rangle(7) \\
a_{a b}^{[4(d, e)]} & =\frac{g_{A}^{2} m_{\pi}}{8 \pi^{4} F_{\pi}^{4}\left(1+m_{\pi} / m_{d}\right)} \sum_{r<s}\left\langle\left(\boldsymbol{t}^{(r)}+\boldsymbol{t}^{(s)}\right) \cdot\left(\boldsymbol{t}^{(\pi)}\right)_{a b} \frac{\vec{q}_{r s} \cdot \vec{\sigma}^{(r)} \vec{q}_{r s} \cdot \vec{\sigma}^{(s)}}{\left(\vec{q}_{r s}{ }^{2}+m_{\pi}^{2}\right)^{3 / 2}}\right\rangle
\end{aligned}
$$


where subscripts $a, b$ are pion isovector indices; $r, s$ label individual nucleons; $\vec{q}_{r s}$ is the momentum transferred between nucleons $r$ and $s$ in their interaction with the pion; $\vec{\sigma}^{(r)}$ and $\boldsymbol{t}^{(r)}$ are the Pauli spin vector and isospin vector of nucleon $r$; and $\left(t_{c}^{(\pi)}\right)_{a b}=-i \epsilon_{a b c}$ is the pion isospin vector. Note that as a result of a partial cancellation between (6) and (7), the integrand in the sum of these averages vanishes for $\vec{q}_{r s} \rightarrow \infty$, which makes the result less sensitive to the behaviour of the nuclear wave function at small internucleon separation.7 To second order in small momenta, the pion-nuclear scattering length is

$$
a_{a b}=\frac{1+m_{\pi} / m_{N}}{1+m_{\pi} / A m_{N}} \sum_{r} a_{a b}^{(r)}+a_{a b}^{[4(a)]}+a_{a b}^{[4(b)]}+a_{a b}^{[4(c)]}+a_{a b}^{[4(d, e)]}
$$

where $a_{a b}^{(r)}$ is the pion scattering length on the $r^{\prime}$ th nucleon.

This all becomes much simpler in two special cases. One is double chargeexchange scattering, $\pi^{+}+N \rightarrow \pi^{-}+N^{\prime}$, where the scattering lengths $a_{a b}^{[r]}$ as well as the corrections (6) and (8) vanish. The other, on which we shall concentrate here, is pion scattering on an isoscalar nucleus. Here $t_{a}^{(r)} t_{b}^{(s)}+$ $t_{a}^{(s)} t_{b}^{(r)}$ may be replaced with $\frac{2}{3} \delta_{a b} \boldsymbol{t}^{(r)} \cdot \boldsymbol{t}^{(s)}$, and Eq. (8) vanishes. More important, the contributions of the nominally leading terms in the pionnucleon scattering lengths vanish, because they involve an expectation value of $\sum_{r} \boldsymbol{t}^{(r)} \cdot \boldsymbol{t}^{(\pi)}$, which vanishes in any isoscalar nucleus. The first term in (9)

\footnotetext{
${ }^{\dagger}$ This cancellation was noted by Robilotta and Wilkin ${ }^{5}$ in the case of pion-deuteron scattering. They used a different definition of the pion field, so their results for diagrams 4(b) and 4(c) were different from (6) and (7), but the sum of their results agrees with what would be found for this process from the sum of (6) and (7).
} 
arises only from " $\sigma$-term" corrections to the pion-nucleon scattering lengths, and is therefore relatively small, making it feasible to compare calculations of the corrections considered here with experimental measurements of the pion-nuclear scattering lengths.

This may be illustrated in the paradigmatic case of pion-deuteron scattering. To evaluate the two-body terms here we need to use isotopic spin invariance to derive the pion-neutron scattering lengths from measured values of the $\pi^{+} p$ and $\pi^{-} p$ scattering lengths. This is not entirely straightforward, because we are interested here in the relatively small corrections to the leading soft-pion results for which $a_{\pi p}+a_{\pi n}=0$, and these corrections arise in part from "sigma terms" proportional to $u$ and $d$ quark masses that do not even approximately conserve isospin. Fortunately to first order in quark masses the isospin violation in the sigma terms affects only processes involving at least one neutral pion, ${ }^{6}$ so that isospin relations can be used to calculate $a_{\pi n}$. This gives the two-body terms in the $\pi-d$ scattering length $\operatorname{as}^{7} \frac{1+m_{\pi} / m_{N}}{1+m_{\pi} / m_{d}}\left[a_{\pi p}+a_{\pi n}\right]=-(0.021 \pm 0.006) m_{\pi}^{-1}$. Shifting to coordinate space, the remaining corrections are given by:

$$
a^{[4 a)]}=-\frac{m_{\pi}^{2}}{\pi^{2} F_{\pi}^{4}\left(1+m_{\pi} / m_{d}\right)} \int_{0}^{\infty} \frac{\left(u^{2}+w^{2}\right)}{r} d r,
$$

and

$$
\begin{aligned}
a^{[4(b, c)]}= & \frac{m_{\pi}^{2} g_{A}^{2}}{3 \pi^{2} F_{\pi}^{4}\left(1+m_{\pi} / m_{d}\right)}\left[\frac{1}{4} \int_{0}^{\infty}\left(u^{2}+w^{2}\right)\left(\frac{1}{r}-\frac{m_{\pi}}{2}\right) e^{-m_{\pi} r} d r\right. \\
& \left.-\int_{0}^{\infty}\left(\frac{u w}{\sqrt{2}}-\frac{w^{2}}{4}\right)\left(\frac{1}{r}+m_{\pi}\right) e^{-m_{\pi} r} d r\right]
\end{aligned}
$$


where $u$ and $w$ are the s-wave and d-wave parts of the deuteron wave function, normalized so that

$$
\int_{0}^{\infty}\left(u^{2}+w^{2}\right) d r=1
$$

The rescattering term (10) [but not (11)] has been previously considered in the books of Eisenberg and Koltun and Ericson and Weise. ${ }^{7}$ Because of the anomalously large radius of the deuteron, this term is considerably larger than the remaining three-body term (11), so it should be calculated including first-order corrections to the pion-nucleon scattering vertices in Figure 4(a). Fortunately these corrections can be taken from the measured values of the scattering lengths. ${ }^{8}$ In this way one finds that ${ }^{7} a^{[4(a)]}=$ $-(0.026 \pm 0.001) m_{\pi}^{-1}$. The remaining three-body terms (11) are calculatedf to be $a^{[4(b, c)]}=-0.0005 m_{\pi}^{-1}$ (mostly arising from the interference between swave and d-wave parts of the wave function), in agreement with the numerical result quoted in reference 5 . This is small compared with the uncertainties in other terms, and so may be neglected here, though this may not be the case for pion scattering on heavier nuclei. This justifies the final theoretical result of reference $7, a_{\pi d}=-(0.050 \pm 0.006) m_{\pi}^{-1}$, which is in good agreement with the experimental value $-(0.056 \pm 0.009) m_{\pi}^{-1}$. Although the use of chiral effective Lagrangians has turned out here only to confirm previous calculations of pion-deuteron scattering as well as nuclear binding, the systematic counting of momentum factors in chiral perturbation theory has proved its value in

\footnotetext{
¥The calculation of the integrals in Eqs. (10) and (11) was carried out by R. C. Mastroleo and U. van Kolck, using the Bonn wave function for the deuteron.
} 
explaining (as previous calculations did not explain) just why it is correct to consider only certain graphs and certain terms in the effective Lagrangian.

I am grateful for discussions with C. Dove, J. Friar, A. Gleeson, C. Ordoñez, U. van Kolck, and J. D. Walecka. 


\section{References}

1. S. Weinberg, Physics Letters B 251 (1990) 288.

2. S. Weinberg, Nuclear Physics B 363 (1991) 3.

3. S. Weinberg, Phys. Rev. Lett. 18 (1967) 188 ; Phys. Rev. 166 (1968) 1568

4. C. Ordoñez and U. van Kolck, Texas preprint UTTG-01-92, to be published in Physics Letters B291 (1992).

5. M. R. Robilotta and C. Wilkin, J. Phys. G: Nucl. Phys., 4 (1978) L115. Also see H. McManus and D. O. Riska, Phys. Lett. 92B (1990) 29.

6. S. Weinberg, in A Festschrift for I. I. Rabi, Transactions of the N. Y. Academy of Sciences 38 (1977) 185.

7. J. M. Eisenberg and D. S. Koltun, Theory of Meson Interactions with Nuclei (Wiley-Interscience, New York, 1980); T. Ericson and W. Weise, Pions and Nuclei (Oxford University Press, Oxford, 1988).

8. V. M. Kolybasov and A. E. Kudryavtsev, Zh. Eksper. Teor. Fiz. (USSR), 63 (1972) 35; Sov. Phys. JETP, 36 (1973) 18. 